\title{
Symmetry breaking in the Anderson-Hubbard model
}

\author{
M.R.M.J. Traa and W.J. Caspers \\ Center for Theoretical Physics, University of Twente, P.O. Box 217, 7500 AE Enschede, \\ The Netherlands
}

Received 25 October 1991

The Anderson-Hubbard $(\mathrm{A}-\mathrm{H})$ model with one or two holes and with periodic boundary conditions on a $4 M \times 4 N$ square lattice is considered. On grounds of an intuitive generalization of Marshall's theorem we split the A-H Hamiltonian $\left(H_{\mathrm{A}-\mathrm{H}}\right)$ into a zeroth order term $\left(H_{0}\right)$ and a perturbation term $\left(H^{\prime}\right)$. With $H_{0}$ we construct unfrustrated states: the zeroth order approximation of the degenerate ground state (GS). The one-hole system has a four-fold symmetry broken $H_{0}$-GS with $k=(\pi / 2, \pm \pi / 2),(-\pi / 2, \pm \pi / 2)$. Group theory shows that this symmetry breaking (SB) may be stable if $H^{\prime}$ is taken into account. For the two-hole system we derive candidates for the $H_{0}$-GS with the corresponding good quantum numbers $k$ and total spin $S$. Here we find no SB or a two-fold SB: again, this result may hold for the complete $H_{\mathrm{A}-\mathrm{H}}$. Second order perturbation calculation possibly describes an effective coupling of two holes.

\section{Introduction}

Since the discovery of high- $T_{c}$ superconducting copper oxides there has been a growing interest in strongly correlated electron systems. Anderson [1] has suggested that the physics of these oxides is contained in the two-dimensional (2D), large- $U$, single-band Hubbard model. In this model doubly occupied (DO) sites are prohibited and the Hubbard Hamiltonian can be transformed to an effective Hamiltonian, called the Anderson-Hubbard (A-H) Hamiltonian [2]. Our main interest is the ground state (GS) of systems on a square lattice with one or two empty sites (holes) and all other sites occupied by one electron. We consider a positive hopping parameter $t$ and a large positive on-site Coulomb repulsion $U$, so that $0<t \ll U$. The $\mathrm{A}-\mathrm{H}$ Hamiltonian reads

$$
\begin{aligned}
& H_{\mathrm{A}-\mathrm{H}}=H_{1}+H_{2}+H_{3}, \\
& H_{1}=-t \sum_{\langle i, j\rangle} \sum_{\sigma}\left(c_{i \sigma}^{\dagger} c_{j \sigma}+\text { H.c. }\right),
\end{aligned}
$$




$$
\begin{aligned}
& H_{2}=2 \frac{t^{2}}{U} \sum_{\langle i, j\rangle} \sum_{\sigma}\left(c_{i \sigma}^{\dagger} c_{i-\sigma} c_{j-\sigma}^{\dagger} c_{j \sigma}-n_{i \sigma} n_{j-\sigma}\right), \\
& H_{3}=-\frac{t^{2}}{U} \sum_{\langle i, j, k\rangle} \sum_{\sigma}\left[\left(c_{i \sigma}^{\dagger} c_{j \sigma} c_{j-\sigma}^{\dagger} c_{k-\sigma}+c_{i \sigma}^{\dagger} n_{j-\sigma} c_{k \sigma}\right)+\text { H.c. }\right] .
\end{aligned}
$$

The symbol $\langle i, j\rangle$ denotes a pair of nearest-neighbour sites $i$ and $j$ and $\langle i, j, k\rangle$ indicates a triple so that $i$ and $j$ as well as $j$ and $k$ are nearest neighbours with $i \neq k$. The $z$-component of an electron spin $s_{z}$ equals $\sigma / 2$ with $\sigma=1$ or -1 $(\hbar=1)$. At half filling, i.e. all sites occupied by one electron, only $H_{2}$ gives a nonzero contribution. It may be shown to be equivalent (apart from an irrelevant additional constant) to the antiferromagnetic (AF) Heisenberg Hamiltonian

$$
H_{\mathrm{Heis}}=J \sum_{\langle i, j\rangle} S_{i} \cdot S_{j}, \quad J=4 \frac{t^{2}}{U},
$$

with the summation over nearest-neighbour sites only and $\boldsymbol{S}_{i}$ denoting the spin operator of an electron on site $i$. The $\mathrm{A}-\mathrm{H}$ model at half filling is a special case of the AF Heisenberg models considered by Marshall [3]. He derived a theorem about the structure of the GS of these AF Heisenberg models. The GS is a linear combination of basis states of a certain space, the so-called Ising configurations (IC) [4]. The product of a phase factor and a positive amplitude represents the coefficients of the IC. The coefficients may be chosen to be real, so the phase factor is 1 or -1 . The theorem predicts the relative phases of the IC uniquely, apart from an overall irrelevant phase. Because of the uniqueness of the relative phases and the fact that all $\mathrm{IC}$, spanning the mentioned space, contribute to the GS, the GS is unique. In general, if the phases of all basis states forming the GS, can be chosen uniquely (apart from an overall phase), the GS is called unfrustrated.

Our approach of the $\mathrm{A}-\mathrm{H}$ model is an intuitive generalization of Marshall's theorem. In section 2 we search for the largest, unfrustrated, linear combination of $\mathrm{IC}$ in the $\mathrm{A}-\mathrm{H}$ model with one hole on a square lattice. We believe that this linear combination gives a good zeroth order approximation of the GS of the system.

\section{The A-H model and frustration}

Consider the $4 M \times 4 N$ square lattice with periodic boundary conditions (PBC) of fig. 1. For convenience we introduce the four square quarter lattices 


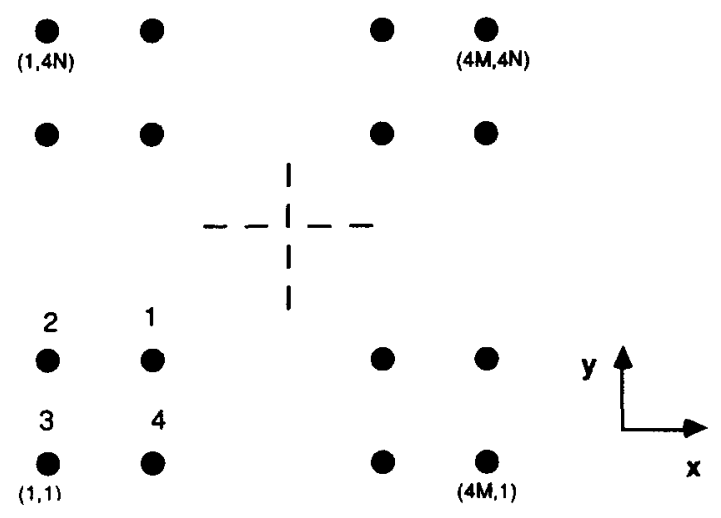

Fig. 1.

(QL) 1, 2, 3, 4 and the two sublattices A resp. B, composed of QL 1 and 3 respectively 2 and 4 . The pseudo-vacuum

$$
|0\rangle=\sum_{\sigma_{1,1} \cdots \sigma_{4 M, 4 N}} A_{\sigma_{1,1} \cdots \sigma_{4 M, 4 N}} c_{1,1 \sigma_{1,1}}^{\dagger} \cdots c_{4 M, 1 \sigma_{4 M, 1}}^{\dagger} c_{1,2 \sigma_{1,2}}^{\dagger} \cdots c_{4 M, 4 N \sigma_{4 M, 4 N}}^{\dagger}|\rangle,
$$

with $\mid>$ denoting the real vacuum, is a Marshall state with $(S, M)=(0,0)$, i.e. $|0\rangle$ is the GS of $H_{2}$. Eq. (2.1) defines the fixed order of creation operators for each IC throughout this article. According to Marshall the sublattice magnetization of an IC determines its phase. We choose the magnetization of sublattice $\mathrm{B}, M_{\mathrm{B}}$, for this purpose,

$$
M_{\mathrm{B}}=\frac{1}{2}\left(M_{+}-M_{-}\right) .
$$

$M_{+}\left(M_{-}\right)$is the number of up (down) spins on sublattice B.

If one takes away from the configuration (2.1) one electron with $s_{z}=\sigma / 2$, one gets a one-hole state. We call the introduced hole a " $\sigma$ " hole. The introduction of a hole will change the amplitudes of the IC in $|0\rangle$. We assume that the phases do not change, because for a fixed position of the hole they are optimal, according to Marshall. We approximate the "rearranged" one-hole state with a " $\sigma$ " hole on site $(m, n)$ by $c_{m, n ; \sigma}|0\rangle$. In general, the $H_{\mathrm{A}-\mathrm{H}^{-}}$GS of the one-hole system is a linear combination of "rearranged" one-hole states and is approximated by

$$
\sum_{m, n} A_{m, n}|(m, n ; \sigma)\rangle=\sum_{m, n} A_{m, n} c_{m, n ; \sigma}|0\rangle
$$

in which the summation is over all sites $(m, n)$. This is a suitable approach, 
because we are primarily interested in the phases and not in the amplitudes of the IC. The intuitive generalization of Marshall's theorem for IC with one hole is expressed by

$$
A_{m^{\prime} n^{\prime}} A_{m, n}\left\langle\left(m^{\prime}, n^{\prime} ; \sigma\right)\left|H_{0}\right|(m, n ; \sigma)\right\rangle \leqslant 0, \quad(m, n) \neq\left(m^{\prime}, n^{\prime}\right),
$$

in which $H_{0}$ is the largest part of $H_{\mathrm{A}-\mathrm{H}}$ so that (2.4) is satisfied with unique phases of the coefficients $A_{m, n}$ and $A_{m^{\prime}, n^{\prime}}$. The corresponding linear combination (2.3) is unfrustrated in this case. We believe that such an unfrustrated linear combination is the main contribution to the GS.

A characteristic of high- $T_{\mathrm{c}}$ superconducting copper oxides is that there is an AF phase for low doping. Without doping, i.e. at half filling, the oxides are AF insulators. The AF background is built up by $H_{2}$. The action of $H_{2}$ is of order $N_{\mathrm{e}}$, with $N_{\mathrm{e}}$ the number of electrons on the lattice. For contributions of $H_{1}$ and $H_{3}$ a pair or a triple of sites with one hole is needed. So for one-hole and two-hole states their action is of order 1 . The amplitude of $H_{1}$ is $t$ and because $t \ll U$ it is much larger than the amplitudes of $H_{2}$ and $H_{3}$, which are of order $t^{2} / U$. In the limit $t / U \rightarrow 0$ only $H_{1}$ is nonzero and the system behaves as a Nagaoka ferromagnet [5]. So the character of $H_{1}$ is ferromagnetic in contrast to $\mathrm{H}_{2}$. Because of the observed antiferromagnetism for low doping, we believe that for large systems the order $N_{\mathrm{e}}$ effect with amplitude $t^{2} / U$ is stronger than the order 1 effect of $H_{1}$ with amplitude $t$. Looking at (2.4) one sees that $H_{0}$ contains at least $\mathrm{H}_{2}$, because states with holes on different sites are orthogonal.

To find the largest possible unfrustrated states obeying (2.4) consider a one-hole state with the hole on QL 1:

$$
\sum_{m, n} A_{2 m, 2 n}|(2 m, 2 n ; \sigma)\rangle
$$

For convenience we split $H_{3}$ into two parts: $H_{3,0}$ and $H_{3,1}$. Under $H_{3,0}$ the hole may be displaced over the vectors $\delta_{0}=( \pm 2,0),(0, \pm 2)$ and under $H_{3,1}$ over $\delta_{1}=(1, \pm 1),(-1, \pm 1)$.

Optimal phases for $H_{3,0}$ may be chosen. To illustrate how the phases are determined, consider only the relevant part of one IC under the action of one term of $H_{3,0}$. Only the resulting phases (not the resulting optimal phases!) are written down. Amplitudes are disregarded. Consider one IC with a " - " hole. The hole is denoted by (-) inside a ket. Then

$$
-H_{3,0}|(-)-+\rangle \longrightarrow|-+(-)\rangle \text {. }
$$

Both $|\mathrm{i}\rangle=|--+\rangle$ and $|\mathrm{f}\rangle=-|-+-\rangle$ occur in $|0\rangle$ with these relative phases. 
Then $\left|i^{\prime}\right\rangle=-|(-)-+\rangle$ occurs in $c_{2 m, 2 n ;-}|0\rangle$ due to the defined order of creation operators in (2.1). Analogously $\left|f^{\prime}\right\rangle=|-+(-)\rangle$ occurs in $c_{2 m+2,2 n ;-}|0\rangle$. Because this process is only one of the contributions of $H_{3,0}$ to $H_{3,0} c_{2 m .2 n ;-}|0\rangle$, this is denoted by

$$
H_{3,0} c_{2 m, 2 n:-}|0\rangle \underset{\mathrm{AO}}{\longrightarrow} c_{2 m+2,2 n ;-}|0\rangle,
$$

in which AO means: among other contributions.

We used the fact that the order of creation and annihilation operators and $M_{\mathrm{B}}$ determine the phase. Proceeding in an analogous way, one may derive

$$
\begin{aligned}
H_{3,0} c_{2 m, 2 n ; \sigma}|0\rangle \longrightarrow & c_{2 m+2,2 n ; \sigma}|0\rangle+c_{2 m-2,2 n ; \sigma}|0\rangle+c_{2 m, 2 n+2 ; \sigma}|0\rangle \\
& +c_{2 m, 2 n-2 ; \sigma}|0\rangle .
\end{aligned}
$$

This gives, for optimal phases, the relation

$$
A_{2 m, 2 n}=(-1)^{m+n}\left|A_{2 m, 2 n}\right| \text {. }
$$

By taking $H_{0}=H_{2}+H_{3,0}$ the lattice is split into four unfrustrated QL. We will show that we cannot add more parts of $H_{\mathrm{A}-\mathrm{H}}$ to this $H_{0}$ without introducing frustration. Taking (2.5) with coefficients (2.9) as starting point, optimal phases for $H_{3,1}$ cannot be chosen.

Example. In (2.5) we have $c_{2 m, 2 n ; \sigma}|0\rangle$ and $-c_{2 m+2,2 n ; \sigma}|0\rangle$. One may show that $H_{3,1}$ acting on these IC gives, among other contributions, $c_{2 m+1,2 n+1 ; \sigma}|0\rangle$ with opposite optimal phases. So $H_{3,1}$ leads to frustration (in second order).

Processes like (2.8) are given in fig. 2. A "+" next to an arrow, denoting the hole's displacement, means that the two IC connected by $H_{3,0}$ or $H_{3,1}$ have opposite optimal phases.

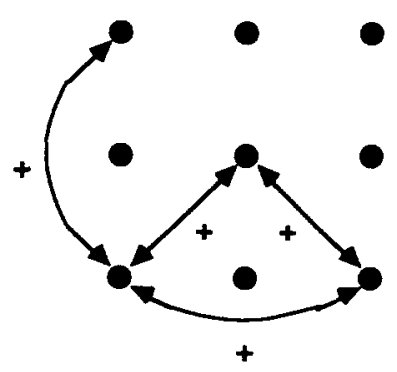

Fig. 2 . 
If one chooses

$$
H_{0}=H_{2}+H_{3,1} \text { and } \quad H^{\prime}=H_{3.0} \text {, }
$$

the hole can move unfrustratedly on a sublattice instead of a QL. But then frustration occurs in first order.

Taking (2.5) with coefficients (2.9) as starting point, optimal phases for $H_{1}$ cannot be chosen either.

Example. In the next four equations, $c_{2 m, 2 n ; \sigma}|0\rangle$ contains the IC in the first column and $-c_{2 m+2,2 n ; \sigma}|0\rangle$ the ones in the third column. The optimal phases of the IC in the second column are the result from the $H_{1}$-process(es), depicted in the same equation. They are denoted by $( \pm)$. The symbol 0 inside a ket denotes a hole.

$$
\begin{gathered}
|0 \sigma-\sigma\rangle \underset{H_{1}}{\longrightarrow}(+)|\sigma 0-\sigma\rangle \overleftarrow{H_{1}}|\sigma-\sigma 0\rangle, \\
-|0-\sigma \sigma\rangle \underset{H_{1}}{\longrightarrow}(-)|-\sigma 0 \sigma\rangle \overleftarrow{H_{1}}-|-\sigma \sigma 0\rangle, \\
|0 \sigma \sigma\rangle \underset{H_{1}}{\longrightarrow}(+)|\sigma 0 \sigma\rangle, \\
(-)|\sigma 0 \sigma\rangle \overleftarrow{H_{1}}-|\sigma \sigma 0\rangle .
\end{gathered}
$$

Eqs. (2.11) and (2.12) show that the resulting IC, (+) $|\sigma 0-\sigma\rangle$ and $(-)|-\sigma 0 \sigma\rangle$, have AF phases for exchanging the opposite spins on the same sublattice. According to Marshall's theorem these IC belong to an excited state instead of the GS. We call this a spin excitation. Eqs. (2.13) and (2.14) lead to destructive interference.

We may conclude from the study of frustration that we get unfrustrated $H_{0}$-eigenstates if we choose

$$
H_{\mathrm{A}-\mathrm{H}}=H_{0}+H^{\prime}, \quad H_{0}=H_{2}+H_{3,0}, \quad H^{\prime}=H_{1}+H_{3,1} .
$$

Because one can start with the hole on four different QL, (2.5) is only one of the four realizations of the $H_{0}$-GS. In section 3 we will prove that the four-fold symmetry breaking of the GS is likely to be stable, even if $H^{\prime}$ is taken into account as a perturbation term on the zeroth order term $H_{0}$. 


\section{Symmetry breaking of the one-hole system}

We accept the $H_{0}$-eigenstate (2.5) with coefficients (2.9) as one of the four realizations of the $H_{0}$-GS, and denote it by $|\mathrm{gr}\rangle_{1}$. Introducing translation operators $T_{x}$ and $T_{y}$ by

$$
T_{x} c_{2 m, 2 n ; \sigma}^{(+)} T_{x}^{-1}=c_{2 m+1,2 n ; \sigma}^{(\dagger)}, \quad T_{y} c_{2 m, 2 n ; \sigma}^{(\dagger)} T_{y}^{-1}=c_{2 m, 2 n+1 ; \sigma}^{(\dagger)},
$$

one may show that

$$
\begin{aligned}
& T_{x}|0\rangle=T_{y}|0\rangle=|0\rangle, \\
& T_{x}^{2}|\mathrm{gr}\rangle_{i}=T_{y}^{2}|\mathrm{gr}\rangle_{i}=-|\mathrm{gr}\rangle_{i}, \quad i=1,2,3,4 .
\end{aligned}
$$

Defining for each fixed pair $(m, n)$

$$
A_{2 m, 2 n}=A_{2 m+1,2 n}=A_{2 m, 2 n+1}=A_{2 m+1,2 n+1},
$$

we get

$$
T_{x}|\mathrm{gr}\rangle_{1}=|\mathrm{gr}\rangle_{2}, \quad T_{y}|\mathrm{gr}\rangle_{1}=|\mathrm{gr}\rangle_{4}, \quad T_{x} T_{y}|\mathrm{gr}\rangle_{1}=|\mathrm{gr}\rangle_{3} .
$$

Combining the $H_{0}$-eigenstates $|\mathrm{gr}\rangle_{i}$ gives

$$
\begin{aligned}
|k\rangle & =|\mathrm{gr}\rangle_{1}+\xi T_{x}|\mathrm{gr}\rangle_{1}+\eta T_{y}|\mathrm{gr}\rangle_{1}+\zeta T_{x} T_{y}|\mathrm{gr}\rangle_{1} \\
& =|\mathrm{gr}\rangle_{1}+\xi|\mathrm{gr}\rangle_{2}+\eta|\mathrm{gr}\rangle_{4}+\zeta|\mathrm{gr}\rangle_{3} .
\end{aligned}
$$

The translational invariance of the system is expressed by

$$
T_{n}|\boldsymbol{k}\rangle=\exp (\mathrm{i} \boldsymbol{k} \cdot \boldsymbol{n})|\boldsymbol{k}\rangle
$$

in which $n=( \pm 1,0)$ or $(0, \pm 1)$. This leads to $k=(\pi / 2, \pm \pi / 2)$ respectively $(-\pi / 2, \pm \pi / 2)$, corresponding with $(\xi, \eta, \zeta)=(-i, \mp i, \mp 1)$ respectively $(i, \mp i, \pm 1)$.

The four states $|k\rangle$ transform according to four inequivalent 1D irreducible representations of the translation group. If one considers the total symmetry group of the square lattice, $G$, we get a $4 D$ irreducible representation of $G$ with the mentioned $\boldsymbol{k}$ forming the star of $\boldsymbol{k}$ [6]. Because $\mathrm{G}$ is not only the symmetry group of $H_{0}$, but also of the complete $H_{\mathrm{A}-\mathrm{H}}$, the $k$ correspond with good quantum numbers (QN) for $H_{\mathrm{A}-\mathrm{H}}$ and states with different $k$ are not 
mixed by $H^{\prime}$. So the four-fold degeneracy of the GS is stable for the transition from $H_{0}$ to $H_{\mathrm{A}-\mathrm{H}}$, at least as long as the shift in energy because of $H^{\prime}$ is not too large. Otherwise the perturbed level may cross one corresponding with different quantum numbers.

The mentioned $\boldsymbol{k}$ are consistent with numerical calculations done by Trug$\operatorname{man}[7]$.

\section{Symmetry breaking of the two-hole system}

Consider a two-hole system on the lattice (with PBC) of fig. 1 with $H_{\mathrm{A}-\mathrm{H}}$ split as in (2.15). One may show that the generalized Marshall theorem leads to unfrustrated states built up with $H_{0}$. We will show that the system with two holes on the same QL is an exception, i.e. is frustrated. We defined the order of creation operators in $|0\rangle$ as in (2.1). Furthermore one has to define a fixed order of the two annihilation operators acting on $|0\rangle$ for a unique determination of the phases in the GS. E.g. for holes on QL 1 and 2 the first annihilation operator corresponds with QL 1 and the second with QL 2. A state with both holes fixed is a Marshall state. Again we assume that only the amplitudes and not the phases are rearranged by introducing two holes in $|0\rangle$.

We approximate the rearranged $H_{0}$-GS, for a two-hole system with e.g. one " $\sigma$ " hole on QL 1 and one " $\sigma$ " hole on QL 2 by

$$
|\mathrm{gr}\rangle_{(1,2)}=\sum_{m, n, p, q} A_{2 m, 2 n ; \sigma \mid 1+2 p, 2 q ; \sigma^{\prime}} c_{2 m, 2 n ; \sigma} c_{1+2 p, 2 q ; \sigma^{\prime}}|0\rangle,
$$

with the relation for optimal phases

$$
A_{2 m, 2 n ; \sigma \mid 1+2 p, 2 q ; \sigma^{\prime}}=(-1)^{m+n+p+q}\left|A_{2 m, 2 n ; \sigma \mid 1+2 p, 2 q ; \sigma^{\prime}}\right|,
$$

The summation in (4.1) is over all sites $(m, n)$ and $(p, q)$.

Ten combinations of two holes are possible. Four combinations with the holes on the same QL and the six combinations of column 1 of table I. The notation $(i, j)$ denotes the defined order of annihilation operators in the corresponding GS.

Table I

\begin{tabular}{lll}
\hline$|(i, j)\rangle$ & $k$ & $S$ \\
\hline$(1,2),(3,4)$ & $(\pi, 0),(\pi, \pi)$ & 0 \\
$(1,4),(2,3)$ & $(0, \pi),(\pi, \pi)$ & 0 \\
$(1,3),(2,4)$ & $(0, \pi),(\pi, 0)$ & 1 \\
\hline
\end{tabular}


If one introduces a "+" and a "-" hole in $|0\rangle$, the condition that equal spin backgrounds have equal phases imposes a consistency condition on the basis states in (4.1). For example, the introduction of a "+" hole on site, say $p_{1}$ on QL 1 and of a "-" hole on site, say $p_{2}$ on QL 2 gives a linear combination of spin configurations with optimal phases, symbolically denoted by

$$
c_{1+} c_{2-}|(+,-)\rangle=|((+),(-))\rangle \text {. }
$$

One gets the same spin configurations by introduction of a "-" hole on $p_{1}$ and a "+" hole on $p_{2}$ :

$$
c_{1-} c_{2+}|(-,+)\rangle=|((-),(+))\rangle \text {. }
$$

For consistency equal spin configurations much have the same phase. If $|(+,-)\rangle$ occurs in $|0\rangle$, then $-|(-,+)\rangle$ occurs in $|0\rangle$ too, according to Marshall. So for QL 1 and 2 we have the consistency condition

$$
\left(c_{1+} c_{2-}-c_{1-} c_{2+}\right)|0\rangle \text {. }
$$

This is a singlet state.

Analogously one finds (for two holes on different sublattices) the singlet states

$$
\left(c_{i+} c_{j-}-c_{i-} c_{j+}\right)|0\rangle \quad \text { for }(i, j)=(3,4),(1,4),(2,3) \text {, }
$$

and (for two holes on the same sublattice) the triplet states

$$
\left(c_{i+} c_{j-}+c_{i-} c_{j+}\right)|0\rangle \quad \text { for }(i, j)=(1,3),(2,4),(1,1),(2,2),(3,3),(4,4) \text {. }
$$

If one introduces two " $\sigma$ " holes on different sublattices, e.g. $c_{1 \sigma} c_{2 \sigma}|0\rangle$, one has a state with $(S, M)=(1,-\sigma)$. According to Lieb and Mattis [8], the overall GS for a two-hole system with the holes on different sublattices and with fixed positions of the holes is a singlet state. A linear combination of such states represents the GS (4.1), which is therefore a singlet state too. Because we are interested in the overall GS, we need not consider the case of two " $\sigma$ " holes on different sublattices. Analogously, the overall GS for a system with two holes on the same sublattice has $S=1$ (see ref. [8]). So for the positions of the holes mentioned in (4.7) the GS may also be realized for two " $\sigma$ " holes. But we will show that for two holes on the same QL frustration always occurs. 
The combinations $(1,3)$ and $(2,4)$ only remain as candidates for the $H_{0}-$ GS with two holes on the same sublattice.

The next example shows that for two holes on the same QL frustration occurs. Interchanging two holes on QL 1 with optimal phases for $H_{3,0}$ gives

$$
\begin{aligned}
& |\mathbf{i}\rangle=c_{2 m .2 n: \sigma} c_{2 m+2.2 n+2 ; \sigma^{\prime}}|0\rangle, \\
& |\mathbf{f}\rangle=-c_{2 m, 2 n: \sigma^{\prime}} c_{2 m+2,2 n+2 ; \sigma}|0\rangle .
\end{aligned}
$$

If $\sigma=\sigma^{\prime}$, then $|\mathrm{i}\rangle$ and $|\mathrm{f}\rangle$ contradict. If $\sigma \neq \sigma^{\prime}$, then (4.9) and (4.10) contradict with (4.7).

As in section 3 we will derive the $\boldsymbol{k}$-values of the $H_{0}$-GS. With $T_{x}$ and $T_{y}$ as in (3.1) one may prove that

$$
T_{x}^{2}|\mathrm{gr}\rangle_{(i, j)}=T_{y}^{2}|\mathrm{gr}\rangle_{(i, j)}=|\mathrm{gr}\rangle_{(i, j)} .
$$

Defining for each fixed quartet $(m, n, p, q)$

$$
\begin{aligned}
A_{2 m, 2 n ; \sigma \mid 2 p+1,2 q ; \sigma^{\prime}} & =A_{2 p+2,2 q ; \sigma^{\prime} \mid 2 m+1,2 n ; \sigma}=-A_{2 p+1,2 q+1 ; \sigma^{\prime} \mid 2 m, 2 n+1 ; \sigma} \\
& =-A_{2 m+1,2 n+1 ; \sigma \mid 2 p+2,2 q+1: \sigma^{\prime}},
\end{aligned}
$$

we get (denoting $|\mathrm{gr}\rangle_{(i, j)}$ by $|(i, j)\rangle$ )

$$
\begin{aligned}
& T_{x}|(1,2)\rangle=-|(1,2)\rangle, \quad T_{y}|(1,2)\rangle=+|(3,4)\rangle, \\
& T_{x} T_{y}|(1,2)\rangle=-|(3,4)\rangle .
\end{aligned}
$$

Combining the $H_{0}$-eigenstates $|(1,2)\rangle$ and $|(3,4)\rangle$ gives

$$
|k\rangle=|(1,2)\rangle+\xi T_{x}|(1,2)\rangle+\eta T_{y}|(1,2)\rangle+\zeta T_{x} T_{y}|(1,2)\rangle .
$$

Translational invariance (cf. (3.7)) leads to

$$
|k\rangle=2|(1,2)\rangle \mp 2|(3,4)\rangle, \quad k=\left\{\begin{array}{l}
(\pi, \pi), \\
(\pi, 0) .
\end{array}\right.
$$

Analogously one finds

$$
\begin{array}{ll}
|k\rangle=2|(1,4)\rangle \pm 2|(2,3)\rangle, & \boldsymbol{k}=\left\{\begin{array}{l}
(0, \pi), \\
(\pi, \pi),
\end{array}\right. \\
|k\rangle=2|(1,3)\rangle \mp 2|(2,4)\rangle, & \boldsymbol{k}=\left\{\begin{array}{l}
(0, \pi) \\
(\pi, 0) .
\end{array}\right.
\end{array}
$$

The results are summarized in table $\mathbf{I}$. 
Because $S^{2}$ and $H_{\mathrm{A}-\mathrm{H}}$ commute, the total spin $S$ is a good QN. As explained in section 3 the $k$ correspond with good QN too. Only $H_{0}$-GS with the same $S$ and $k$ are mixed by the perturbation term $H^{\prime}$. Looking at table $\mathrm{I}$, one sees that our $k$ are consistent with the $k$ of the GS of the two-hole system, $k=(\pi, \pi)$, numerically found by Trugman [7].

Our idea is that this mixing of $H_{0}$-GS leads to an effective coupling of two holes in second order perturbation calculation. Eqs. (2.11) and (2.12) show that for certain IC in a $H_{0}$-GS two steps with $H_{1}$ give the same phases as one step with $H_{3,0}$. But the intermediate state, reached after one step with $H_{1}$, has a spin excitation. For a one-hole state the excitation is removed by another step of the hole with $H_{1}$, parallel or antiparallel with the first one. For a two-hole state one can imagine that the second hole makes the second step with $H_{1}$, thereby removing the excitation. The second step need not be parallel or antiparallel with the first one. For example, for $S=0$ and $k=(\pi, \pi)$ one may have the transition from $(1,2)$ to $(2,3)$ via the intermediate state $(2,2)$. So, two holes which interact properly have a larger freedom of movement.

The idea that a hole moves primarily on an "unfrustrated part of the lattice" and that this may lead to symmetry breaking is supported by the exact calculation of the $\mathrm{H}_{\mathrm{A}-\mathrm{H}}$-GS of the 1D system: a ring with one hole, two electrons with spin up and one with spin down. The exact GS is two-fold degenerate. Each realization is built up of two parts. The first part is an unfrustrated linear combination of IC with the hole on one sublattice. The phases are optimal for $H_{0}=H_{2}+H_{3}$. In a 1D system $H_{3}=H_{3,0}$. The second part is built up of IC with the hole on the other sublattice. The matrix elements of $H_{1}$ between the IC with their corresponding coefficients of the first and the second part of a realization of the GS are all negative or zero, i.e. for $H_{1}$-processes the phases are optimal. But the phases of the IC of the second part are anti-optimal for $H_{0}$. So the IC of the second part correspond with a spin excitation. One may show that the chance to find the hole on the "unfrustrated sublattice" is larger than to find it on the "frustrated sublattice".

Note that 1D and 2D frustration are different. In 1D, $H_{3,1}$ does not exist. Furthermore one does not have the benefit of the order $\mathrm{N}_{\mathrm{e}}$ effect of $\mathrm{H}_{2}$ in such a small system as the mentioned ring. So, although the ring gives supporting results, the need for calculations on $2 \mathrm{D}$ systems is clear.

\section{References}

[1] P.W. Anderson, Science 235 (1987) 1196.

[2] C. Gros, R. Joynt and T.M. Rice, Phys. Rev. B 36 (1987) 381. 
[3] W. Marshall, Proc. R. Soc. A 232 (1955) 48.

[4] P.L. Iske and W.J. Caspers, Physica A 146 (1987) 151.

[5] Y. Nagaoka, Solid State Commun. 3 (1965) 409; Phys. Rev. 147 (1966) 392.

[6] M. Tinkham, Group Theory and Quantum Mechanics (McGraw-Hill, New York, 1964).

[7] S.A. Trugman, Phys. Rev. B 37 (1988) 1597.

[8] E. Lieb and D. Mattis, J. Math. Phys. 3 (1962) 749. 